\title{
VAC_15 - Production and evaluation of a vaccine formulation composed by chimeric protein with protective potential action against Leishmania infantum
}

Bianca de Oliveira ${ }^{1 *}$; Natália Salazar de Castro $^{1}$; Bárbara R. B. V. Azevedo ${ }^{1}$; Júlia T. Castro ${ }^{2}$; Frederico C. Nascimento ${ }^{1}$; Ana Paula Salles Moura Fernandes ${ }^{1}$; Ricardo Tostes Gazzinelli ${ }^{3}$.

${ }^{1}$ UFMG - Universidade Federal de Minas Gerais;

${ }^{2}$ USP - Universidade de São Paulo;

${ }^{3}$ Fiocruz/CPqRR.

Introduction: Human visceral leishmaniasis (HVL) ranks second in mortality rates among tropical infectious diseases. Therefore, there is an urgent need of a prophylactic vaccine for HVL. Among the antigens candidate, different studies show that Leishmania amastigote 2 (A2) protein is immunogenic and this protein is commercially available in a vaccine for canine visceral leishmaniasis. However, the presence of saponin as adjuvant makes its formulation improper to be used in humans. Thus, towards vaccination in humans, it was proposed to develop a recombinant chimeric protein with the presence (rDTL4_tag) and the absence (rDTL4) of the histidine tag. This antigen contains a fragment of A2 and, combined with specific adjuvants, may be used as a strategy for vaccination against HVL.

Objective: Express and purify the rDTL4_tag and rDTL4 protein, as well as explore the vaccine potential of rDTL4_tag by testing it in combination with different immunological adjuvants already approved for human vaccination.

Methodology: 1) The rDTL4_tag and rDTL4 proteins were expressed in E. coli BL21 (DE3) bacteria and purified, respectively, by affinity chromatography and by two ion exchange chromatographies in the Akta prime (GE) system. The purified fraction of the protein rDTL4_tag was loaded onto a ToxinEraserTM (GenScript) column to remove endotoxins. 2) Female Balb/c mice were immunized with rDTL4_tag antigen associated with different adjuvants (Alumem/CPG, Poly(I:C) or AddaVax). It should be noted that the formulation containing recombinant protein $\mathrm{A} 2$ (rA2) was established as a positive control and, as negative control, saline or adjuvants separetely. Therefore, the animals were evaluated for protection given against the challenge with $1 \times 10^{7} \mathrm{~L}$. infantum promastigotes. After the challenge, the number of viable parasites per milligram of the infected organs was determined using limiting dilution tests. 3) For the assessment of immunogenicity, an ELISA test was used to measure the levels of total IgG, IgG1 and IgG2a specific for the recombinant proteins as well as to measure the levels of IFN- $\gamma$ and IL-10 in the culture of splenocytes stimulated with rDTL4 tag and rA2.

Results: The outcomes of the purification of rDTL4_tag and rDTL4 were satisfactory, presenting a high degree of purity.The immunized animals with the formulations containing rDTL4 tag showed stronger cellular immune response than the control groups, as revealed by the increased levels of IFN- $\gamma$. In addition, the rDTL4_tag protein associated with the Poly(I:C) adjuvant induced robust production of antigen-specific total $\operatorname{IgG}, \operatorname{IgG1}$ and $\operatorname{IgG} 2$ a. Furthermore, this group still showed a superior protection against infection, as shown by the decrease of tissue parasitism.

Conclusion: The success of the rDTL4_tag antigen makes it a promising candidate for vaccine formulation. Thus, the vaccination strategy explored reveals promising alternatives for the development of an effective vaccine against HVL, aiming at the transposition of the rDTL4 protein for clinical trials in humans.

Keywords: Human visceral leishmaniasis; Vaccine; Recombinant protein 